\title{
Efeito do fotoperíodo sobre o ciclo de vida e a morfometria da concha de Dysopeas muibum (Mollusca, Subulinidae) em condições de laboratório
}

\author{
Vinícius J. Pilate ${ }^{1,2}$, Lidiane C. Silva ${ }^{2,3}$ \& Elisabeth C. A. Bessa ${ }^{2,4}$
}

\footnotetext{
1. Programa de Pós-graduação em Ciências Biológicas - Comportamento e Biologia Animal, Universidade Federal de Juiz de Fora, Juiz de Fora, MG, Brasil. (viniciuspilate@gmail.com) 2. Núcleo de Malacologia da Universidade Federal de Juiz de Fora, Juiz de Fora, MG, Brasil.

3. Programa de Pós-graduação em Ciências Veterinárias - Parasitologia Veterinária, Universidade Federal Rural do Rio de Janeiro, Seropédica, RJ, Brasil (lhybio@yahoo.com.br)

4. Departamento de Zoologia, Instituto de Ciências Biológicas, Universidade Federal de Juiz de Fora, Juiz de Fora, MG, Brasil (elisabeth.bessa@ufjf.edu.br)
}

\begin{abstract}
Effect of photoperiod on the life cycle and shell morphology of Dysopeas muibum (Mollusca, Subulinidae) under laboratory conditions This study aimed to evaluate the effect of photoperiod on the life cycle and shell morphology of Dysopeas muibum Marcus \& Marcus, 1968 under laboratory conditions. To this end, we conducted monitoring growth of the shell, the release of puppies and mortality of 40 specimens undergo natural conditions of temperature, relative humidity and photoperiod, and 80 undergo controlled conditions ( 40 with long days, and 40 with short days), from birth to 180 days, when the measures of the shell were taken. It was found that the photoperiod had no effect on the indeterminate growth, the relationship between reproduction and growth, iteroparidade and low mortality before and after sexual maturity. The controlled conditions of temperature, relative humidity and photoperiod favored growth, conchiliomorphometrics values higher and fast reach sexual maturity, while the decrease in day length favored fecundity.
\end{abstract}

KEYWORDS. Luminosity, reproduction, conchiliomorphometry, terrestrial mollusc.

RESUMO. Neste estudo teve-se como objetivo avaliar o efeito do fotoperíodo sobre o ciclo de vida e a morfometria da concha de Dysopeas muibum Marcus \& Marcus, 1968 em condições de laboratório. Para tal, foram acompanhados o crescimento da concha, a liberação de filhotes e a mortalidade de 40 espécimes submetidos a condições naturais de temperatura, umidade relativa do ar e fotoperíodo, e 80 submetidos a condições controladas (40 com dias longos e $40 \mathrm{com}$ dias curtos), desde o nascimento até 180 dias de vida, quando foram aferidas as medidas da concha. Verificou-se que o fotoperíodo não teve efeito sobre o padrão de crescimento indeterminado, a relação entre reprodução e crescimento, a iteroparidade e a baixa mortalidade antes e após a maturidade sexual. As condições controladas de temperatura, umidade relativa do ar e fotoperíodo favoreceram o crescimento, maiores valores conquiliomorfométricos e o rápido alcance da maturidade sexual, enquanto a diminuição do comprimento do dia favoreceu a fecundidade.

PALAVRAS-CHAVE. Luminosidade, reprodução, conquiliomorfometria, molusco terrestre.

O ciclo de vida e a morfologia dos moluscos terrestres são fortemente influenciados por alterações nos fatores abióticos, como temperatura, pluviosidade, umidade, substrato e fotoperíodo (Lüsis, 1966; Dimitrieva, 1975; WAREING \& BAILEY, 1985; Gomot et al., 1989; Gomot, 1990; South, 1992; Asami, 1999; D’Ávila et al., 2004; D'Ávila \& Bessa, 2005a,b,c; HAUSDORF, 2006; ANDERSON et al., 2007; UDAKA et al., 2007). Mudanças sazonais em alguns destes fatores podem regular as taxas de crescimento, o desenvolvimento, a sobrevivência e o ciclo reprodutivo de gastrópodes terrestres, incluindo a gametogênese, a corte e a cópula, principalmente pelas mesmas atuarem no controle hormonal e na conversão de reservas energéticas (WAYNE, 2001; Garcia \& Pinheiro, 2007; Udaka et al., 2007).

Em razão do endemismo das espécies, da degradação de habitats e da introdução de espécies exóticas, muitas espécies de pulmonados terrestres podem estar sendo extintas antes mesmo de serem descritas, o que evidencia a necessidade de estudos biológicos e morfológicos, visando auxiliar o planejamento de estratégias de manejo e conservação (BACKElJAU et al., 2001; TELEs \& Fontes, 2002).

Os micromoluscos terrestres Dysopeas muibum Marcus \& Marcus, 1968 integram a família Subulinidae e são nativos do continente americano, ocorrendo em locais protegidos e úmidos. No Brasil a espécie foi registrada nas cidades de São Paulo, SP e Juiz de Fora, MG (Simone, 2006; Pilate et al., 2012). Até o momento, foram realizados com esta espécie estudos de comportamento (PILATE et al., 2012; 2013), de ciclo de vida e de conquiliomorfometria em condições de laboratório (Pilate et al., 2013) e de campo (Mota \& Almeida, 2012), e do efeito do isolamento sobre aspectos biológicos e morfológicos (Pilate et al., 2013). Ainda não foram conduzidos trabalhos que esclareçam a influência de fatores abióticos sobre a biologia e a morfologia de D. muibum.

O objetivo do estudo foi avaliar o efeito do fotoperíodo sobre o ciclo de vida e a morfometria da concha de D. muibum em condições de laboratório.

\section{MATERIAL E MÉTODOS}

O estudo foi feito com base em 120 espécimes de D. muibum recém-nascidos, obtidos a partir de criações matrizes de laboratório formadas por espécimes coletados em uma horta do bairro Parque Independência, município de Juiz de Fora, Minas Gerais. Estes foram organizados em 12 grupos, cada um dos quais contendo 10 espécimes, mantidos desde o nascimento até 180 dias de vida, entre os meses de março a setembro de 2011. 
Cada grupo foi incluído em um terrário plástico transparente de $1 \mathrm{~L}$ ( $14 \mathrm{~cm}$ de diâmetro e $9 \mathrm{~cm}$ de altura), fechado com tecido de algodão e elástico, contendo $200 \mathrm{~g}$ (3 $\mathrm{cm}$ de altura $)$ de terra vegetal comercial esterilizada $\left(120^{\circ} \mathrm{C}\right.$ por uma hora) como substrato, umedecida a intervalos de dois dias, com $10 \mathrm{~mL}$ de água de torneira.

Os espécimes foram alimentados ad libitum com ração para frangos de corte peneirada e enriquecida com carbonato de cálcio (na proporção de 3:1), oferecida em discos plásticos transparentes de $4 \mathrm{~cm}$ de diâmetro (um disco por terrário) (BESSA \& ARAÚJo, 1995a,b).

Foram formados três grupos experimentais: (1) Controle: 40 espécimes (quatro grupos, 10 moluscos por grupo) mantidos sob condições naturais de temperatura, umidade relativa do ar e fotoperíodo, as temperaturas mínima e máxima e a umidade relativa do ar registradas diariamente com termômetro de máxima e de mínima e termo-higrômetro de bulbo seco e úmido, respectivamente, sendo a média dos registros de temperatura de 20,30 \pm $2,24^{\circ} \mathrm{C}$ e de umidade relativa do ar, de $78,90 \pm 10,15 \%$; (2) Dia longo: 40 espécimes (quatro grupos, 10 moluscos por grupo) mantidos em estufa para B.O.D., a $22^{\circ} \mathrm{C}$, com umidade relativa do ar de aproximadamente $80 \%$ e fotoperíodo de 14 horas diárias de luz e 10 de escuro e (3) Dia curto: 40 espécimes (quatro grupos, 10 moluscos por grupo) mantidos em estufa para B.O.D., a $22^{\circ} \mathrm{C}$, com umidade relativa do ar de aproximadamente $80 \%$ e fotoperíodo de 10 horas diárias de luz e 14 de escuro.

A intervalos de 15 dias, a partir do primeiro dia do experimento até 180 dias de vida, foi registrada a medida do comprimento da concha dos moluscos dos três grupos experimentais para estabelecer o crescimento médio $(\mathrm{mm})$ e a taxa de crescimento (mm por dia) durante o ciclo de vida. Os moluscos que atingiram 180 dias foram destinados ao estudo da conquiliomorfometria. Foram obtidas as seguintes medidas dos três grupos experimentais, adaptadas da metodologia usada por CHIU et al. (2002): comprimento da concha, largura da concha, comprimento da abertura, largura da abertura, comprimento da volta corporal, comprimento da penúltima volta e comprimento da espira. Também foi determinado o número de voltas da concha. Com os valores destas variáveis lineares, foram calculadas razões, utilizadas como descritores da forma da concha: comprimento da concha/largura da concha, comprimento da volta corporal/comprimento da penúltima volta, comprimento da volta corporal/largura da concha, comprimento da abertura/largura da abertura e comprimento da espira/comprimento da volta corporal. Medições foram feitas com o uso de um paquímetro.

O aparecimento de filhotes nos terrários, verificado através de observações diárias, foi o parâmetro utilizado para a constatação do tempo para o alcance da maturidade sexual. A partir desse dia até 180 dias, foram realizadas observações diárias para a verificação do número de eventos reprodutivos realizados, do número de filhotes por evento reprodutivo e do intervalo entre eventos reprodutivos. Um evento reprodutivo foi caracterizado como um encontro de filhotes em um terrário, sendo os mesmos quantificados, retirados e transferidos para as criações matrizes. Com base nesses dados, foi calculada a fecundidade (número médio de filhotes produzidos por evento reprodutivo e número médio de filhotes produzidos por molusco parental). $\mathrm{O}$ número de filhotes por indivíduo foi quantificado dividindose o número de filhotes em cada evento reprodutivo pelo número total de moluscos do grupo, por não ser possível saber quais moluscos do grupo se reproduziram e o número de filhotes que cada um procriou.

Para avaliar a taxa de mortalidade total, antes e após a maturidade sexual dos moluscos dos três grupos experimentais, foram realizadas observações diárias e cada morte observada foi registrada. Animais mortos foram retirados do terrário.

Aplicou-se a análise de variância Kruskal-Wallis $(\mathrm{H})$, seguida pelo teste Student-Newman-Keuls (t) para a comparação das frequências médias dos parâmetros de crescimento, conquiliomorfometria, reprodução e mortalidade dos moluscos dos três grupos experimentais. Para correlacionar o número médio de filhotes e o comprimento médio da concha ao longo dos 180 dias de experimento, foi utilizado o teste de Regressão Linear Simples (F). Para verificar a possível separação destes grupos, através de similaridade entre os padrões conquiliomorfométricos, foram calculados os valores da distância euclidiana e realizadas as análises de agrupamentos pelo método de agregação Ward e discriminante. Previamente, os valores das variáveis lineares das conchas foram submetidos à transformação logarítmica $\left(\log _{10}\right)$ para minimizar os desvios da normalidade nos valores brutos. As análises foram realizadas no programa BioEstat 5.0 e foi adotado o índice de significância de 0,05 .

\section{RESULTADOS}

O comprimento médio dos moluscos dos grupos Controle, Dia longo e Dia curto ao final do experimento foi de 7,26 $\pm 0,59 \mathrm{~mm}, 9,48 \pm 0,90 \mathrm{~mm}$ e 10,03 $\pm 0,72 \mathrm{~mm}$, respectivamente, havendo diferença significativa entre estes $(\mathrm{H}=57,99 ; \mathrm{p}=0)$. Os moluscos dos grupos Controle e Dia longo alcançaram valores significativamente maiores no comprimento da concha com relação ao grupo Dia curto nos 15 primeiros dias experimento $(H=9,97 ; p=0,01)$. O grupo Controle, no entanto, apresentou valores significativamente menores dessa medida em relação aos grupos Dia longo e Dia curto no início da atividade reprodutiva $(\mathrm{H}=34,89$; $\mathrm{p}=0)$ e no final do experimento $(\mathrm{H}=57,99 ; \mathrm{p}=0)$ (Fig. 1).

Foi verificada diferença significativa no comprimento da concha entre moluscos dos grupos Controle, Dia longo e Dia curto em todas as idades. Os moluscos do grupo Controle apresentaram valores significativamente menores do comprimento da concha em todas as idades, quando comparados aos moluscos do grupo Dia longo, com exceção das idades de $15(\mathrm{t}=8,01 ; \mathrm{p}=0,30)$ e 45 dias $(\mathrm{t}=9,03 ; \mathrm{p}=0,23)$ que não diferiram entre estes dois grupos e da idade de 30 dias, onde apresentaram valores significativamente maiores 
$(t=20,30 ; p=0,01)$. Quando comparados aos moluscos do grupo Dia curto, apresentaram valores significativamente menores em todas as idades, com exceção da idade de 30 dias que não diferiu entre estes dois grupos $(t=11,02$; $\mathrm{p}=0,15$ ) e da idade de 15 dias, onde apresentaram valores significativamente maiores $(\mathrm{t}=23,55 ; \mathrm{p}<0,01)$. Os indivíduos do grupo Dia longo apresentaram valores significativamente menores do comprimento da concha nas idades de 150 dias $(\mathrm{t}=16,50 ; \mathrm{p}=0,02)$ e 165 dias $(\mathrm{t}=15,32 ; \mathrm{p}=0,02)$, quando comparados aos indivíduos do grupo Dia curto. Porém, apresentaram valores significativamente maiores na idade de 15 dias $(\mathrm{t}=15,54 ; \mathrm{p}=0,04)$.

Observou-se que dos 45 dias até o alcance da maturidade sexual, os moluscos dos grupos Dia longo e Dia curto apresentaram um ritmo de crescimento mais rápido com relação ao grupo Controle. Após esse período, a taxa de crescimento nos três grupos passou a ser semelhante no decorrer do tempo. Porém, tomando todo o período analisado, a taxa de crescimento dos moluscos dos três grupos não diferiu estatisticamente $(\mathrm{H}=0,92$; $\mathrm{p}=0,63$ ), demonstrando que o fotoperíodo não interfere nesse parâmetro. Dysopeas muibum apresentou padrão de crescimento indeterminado nos três tratamentos, uma vez que depois de maduros, a taxa de crescimento diminui nos moluscos dos grupos Controle $(t=4,83 ; p=0,02)$, Dia longo $(t=6,00 ; p=0,01)$ e Dia curto $(t=5,66 ; p=0,01)$.

Em relação à conquiliomorfometria, foi demonstrada existência de diferença significativa em todas as variáveis lineares das conchas entre moluscos dos grupos Controle, Dia curto e Dia longo. Em relação às razões entre essas variáveis, foi demonstrada existência de diferença significativa nas razões $\mathrm{CC} / \mathrm{LC}, \mathrm{CVC} / \mathrm{CPV}$ e $\mathrm{CE} / \mathrm{CVC}$, o que não aconteceu em CVC/LC $(\mathrm{H}=1,41 ; \mathrm{p}=0,49)$ e CA/ LA $(\mathrm{H}=0,77 ; \mathrm{p}=0,68)(\mathrm{Tab} . \mathrm{I})$.

Os moluscos do grupo Controle apresentaram valores significativamente menores de todas as variáveis quando comparados aos moluscos do grupo Dia curto, e de todas as variáveis, com exceção de $\mathrm{CPV}$, que não foi significativamente diferente $(\mathrm{H}=2,17 ; \mathrm{p}=0,74)$, quando comparados aos moluscos do grupo Dia longo. Os moluscos do grupo Dia curto exibiram valores significativamente maiores nas variáveis $\operatorname{CPV}(\mathrm{t}=24,24 ; \mathrm{p}<0,01)$ e $\mathrm{CE}$ $(t=14,33 ; p=0,03)$ do que os do grupo Dia longo (Tab. I).

Em relação às razões entre essas variáveis, os moluscos do grupo Controle mostraram valores significativamente mais baixos na razão $\mathrm{CC} / \mathrm{LC}$, quando comparados aos grupos Dia curto $(\mathrm{t}=34,31 ; \mathrm{p}<0,01)$ e Dia longo ( $t=34,09 ; p<0,01)$, o mesmo ocorrendo na razão $\mathrm{CE} /$ CVC, ao ser feita a comparação com os grupos Dia curto $(t=39,90 ; p<0,01)$ e Dia longo ( $t=29,42 ; p<0,01)$. Também apresentaram valores significativamente mais baixos na razão $\mathrm{CVC} / \mathrm{CPV}$, quando comparados ao grupo Dia longo $(t=23,74 ; p<0,01)$, o mesmo ocorrendo com os moluscos do grupo Dia curto $(t=14,92 ; p=0,02)($ Tab. I).

Com base nesses resultados, de maneira geral, o grupo Dia curto possui os maiores valores das variáveis lineares da concha e das razões entre essas variáveis, o grupo Controle, os menores valores e o grupo Dia longo, valores intermediários. Tal relação foi confirmada pelas relações de distância observadas por meio das análises de distância Euclidiana, de agrupamentos e discriminante.

A análise de distância Euclidiana aplicada às variáveis lineares das conchas dos grupos Controle, Dia curto e Dia longo revelou maior distância entre os grupos Controle e Dia curto $(6,28)$ e menor distância entre os grupos Dia curto e Dia longo $(2,57)$. Na análise de agrupamentos, os moluscos dos grupos Dia curto e Dia longo foram agrupados com $40 \%$ de distância, enquanto os do grupo Controle formaram um grupo externo com 100\% de distância desse agrupamento (Fig. 2).

A análise discriminante aplicada às variáveis lineares dos grupos Controle, Dia curto e Dia longo demonstrou que os padrões conquiliomorfométricos, apesar de formarem



Fig. 1. Crescimento médio de Dysopeas muibum Marcus \& Marcus, 1968 dos grupos experimentais Controle, Dia longo e Dia curto, durante 180 dias de vida (setas indicam idades de alcance da maturidade sexual (seta preta, Controle; seta branca, Dia longo; seta cinza, Dia curto).

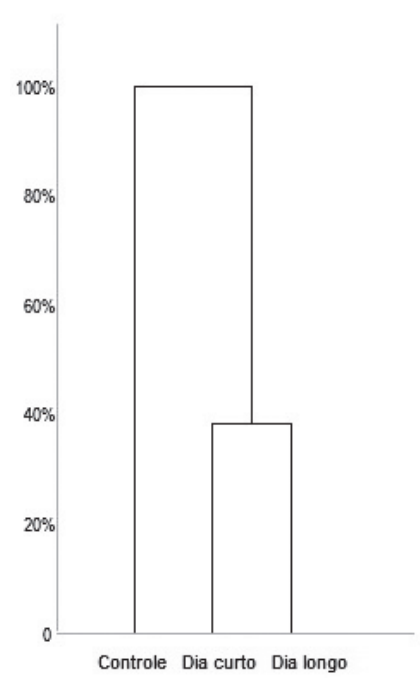

Fig. 2. Análise de agrupamentos aplicada às variáveis lineares da concha de Dysopeas muibum Marcus \& Marcus, 1968 dos grupos experimentais Controle, Dia curto e Dia longo, com idade de 180 dias de vida. 
Tab. I. Média, desvio padrão e coeficiente de variação das variáveis lineares da concha (mm) de Dysopeas muibum Marcus \& Marcus, 1968 dos grupos experimentais Controle, Dia curto e Dia longo, com idade de 180 dias de vida, e razões entre essas variáveis. Letras diferentes indicam diferença significativa entre os grupos experimentais para a variável linear/razão analisada $(\mathrm{p}<0,05)(\mathrm{CV}$, coeficiente de variação; $\mathrm{CA}$, comprimento da abertura; CC, comprimento da concha; $\mathrm{CE}$, comprimento da espira; $\mathrm{CPV}$, comprimento da penúltima volta; CVC, comprimento da volta corporal; DP, desvio padrão; LA, largura da abertura; LC, largura da concha; NV, número de voltas; CA/LA, razão entre comprimento da abertura e largura da abertura; CC/LC, razão entre comprimento da concha e largura da concha; $\mathrm{CE} / \mathrm{CVC}$, razão entre comprimento da espira e comprimento da volta corporal; CVC/CPV, razão entre comprimento da volta corporal e comprimento da penúltima volta; CVC/LC, razão entre comprimento da volta corporal e largura da concha).



grupos com componentes dispersos, mostraram variação. Os moluscos do grupo Controle ficaram pouco relacionados com os do grupo Dia curto, não havendo componentes sobrepostos no gráfico. Já os moluscos do grupo Dia longo, apesar de formarem um grupo separado, se mantiveram relacionados com os outros dois grupos, com muitos componentes sobrepondo-se no gráfico com o grupo Dia curto, mas pouca sobreposição com o grupo Controle, confirmando as relações obtidas com as análises de distância Euclidiana e de agrupamentos (Fig. 3).

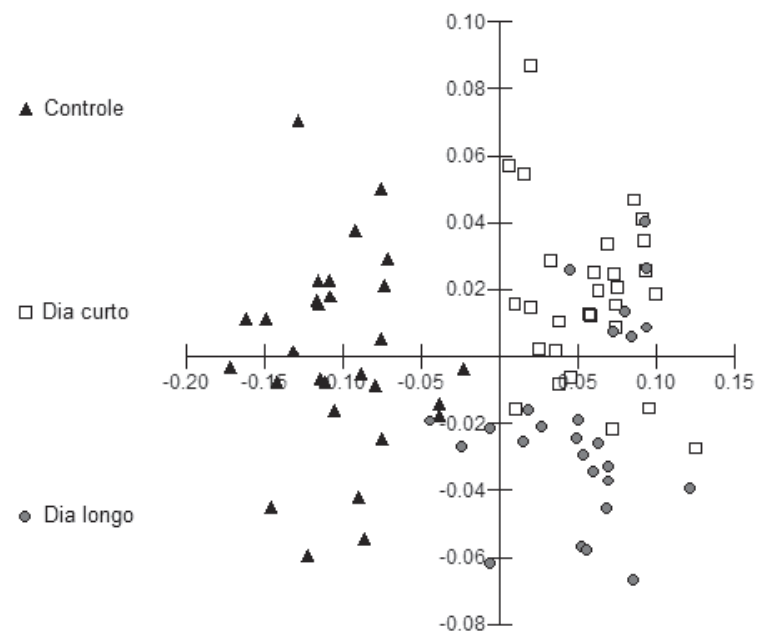

Fig. 3. Análise discriminante aplicada às variáveis lineares da concha de Dysopeas muibum Marcus \& Marcus, 1968 dos grupos experimentais Controle, Dia curto e Dia longo, com idade de 180 dias de vida.
Com relação à reprodução, o tempo para alcançar a maturidade sexual de moluscos do grupo Controle foi de $89 \pm 6,93$ dias. Esse período foi significativamente menor para os moluscos do grupo Dia longo, $75 \pm 0$ dias $(\mathrm{t}=6,00$; $\mathrm{p}=0,02)$, assim como para os do grupo Dia curto, $75 \pm 0$ dias $(t=6,00 ; p=0,02)$, o que evidencia que a alteração do fotoperíodo causou diminuição significativa do tempo para início da liberação de filhotes. No entanto, esse período não foi significativamente diferente entre os grupos Dia longo e Dia curto $(t=0,00 ; p=1,00)$.

Os indivíduos do grupo Controle realizaram 16,25 $\pm 2,87$ eventos reprodutivos, obtendo um total de 295 filhotes, enquanto os do grupo Dia longo realizaram 20,75 $\pm 0,96$ eventos, totalizando 787 filhotes e os moluscos do grupo Dia curto, $23 \pm 1,15$, com 983 filhotes. Houve diferença significativa entre os três tratamentos com relação ao número de eventos reprodutivos $(\mathrm{H}=8,97 ; \mathrm{p}=0,01)$ e o número total de filhotes produzidos $(\mathrm{H}=8,00 ; \mathrm{p}=0,02)$. Quando os tratamentos foram comparados dois a dois, houve diferença significativa no número de eventos reprodutivos entre Controle e Dia curto $(\mathrm{t}=7,50 ; \mathrm{p}<0,01)$, o que não ocorreu entre Controle e Dia longo $(t=3,75 ; p=0,14)$ e entre Dia longo e Dia curto $(t=3,75 ; p=0,14)$. Da mesma forma, com relação ao número total de filhotes produzidos, Controle e Dia curto diferiram $(\mathrm{t}=7,00 ; \mathrm{p}=0,01)$, o que não ocorreu entre Controle e Dia longo $(\mathrm{t}=5,00 ; \mathrm{p}=0,05)$ e entre Dia longo e Dia curto $(t=2,00 ; p=0,43)$. O intervalo entre eventos reprodutivos foi significativamente diferente entre os grupos Controle (5,85 $\pm 3,45$ dias), Dia longo $(5,27 \pm 3,05$ dias $)$ e Dia curto $(4,77 \pm 1,98$ dias $)(\mathrm{H}=7,09$; $\mathrm{p}=0,03)$. Quando comparados dois a dois, houve diferença significativa entre Controle e Dia curto $(t=6,50 ; p=0,01), o$ 
que não se deu entre Controle e Dia longo $(t=1,75 ; p=0,49)$ e entre Dia longo e Dia curto $(t=4,75 ; \mathrm{p}=0,06)$.

Os moluscos do grupo Controle obtiveram 4,60 $\pm 1,26$ filhotes por evento reprodutivo e 7,38 $\pm 2,08$ por molusco parental, enquanto os moluscos do grupo Dia longo obtiveram $9,51 \pm 0,74$ por evento e $19,68 \pm 0,75$ por molusco, e os moluscos do grupo Dia curto, 10,63 $\pm 2,06$ filhotes por evento e 24,58 $\pm 5,69$ filhotes por molusco. A fecundidade de $D$. muibum observada nos três tratamentos diferiu, sendo muito ampliada pela redução do comprimento do dia, tanto no número de filhotes por evento reprodutivo $(\mathrm{H}=8,00 ; \mathrm{p}=0,02)$, quanto no número de filhotes por molusco $(\mathrm{H}=8,00 ; \mathrm{p}=0,02)$. Quando comparados dois a dois, houve diferença significativa no número de filhotes por evento reprodutivo entre Controle e Dia curto $(t=7,00$; $\mathrm{p}=0,01)$, o que não ocorreu entre Controle e Dia longo $(t=5,00 ; p=0,05)$ e entre Dia longo e Dia curto $(t=2,00$; $\mathrm{p}=0,43$ ), assim como no número de filhotes por molusco parental entre Controle e Dia curto $(t=7,00 ; p=0,01)$, o que não aconteceu entre Controle e Dia longo $(\mathrm{t}=5,00 ; \mathrm{p}=0,05)$ e entre Dia longo e Dia curto $(t=2,00 ; p=0,43)$.

O teste de Regressão Linear Simples revelou ser significativa a relação entre o crescimento e a reprodução, representados respectivamente pelo comprimento médio da concha e pelo número médio de filhotes de moluscos dos grupos Controle $(y=-20,97+0,91 x ; F=10,74 ; p=0,01$; $\left.\mathrm{R}^{2}=0,47\right)$, Dia longo $(\mathrm{y}=-31,98+1,74 \mathrm{x} ; \mathrm{F}=14,09 ; \mathrm{p}<0,01$; $\left.\mathrm{R}^{2}=0,54\right)$ e Dia curto $(\mathrm{y}=-46,43+1,16 \mathrm{x} ; \mathrm{F}=8,29 ; \mathrm{p}=0,02$; $\mathrm{R}^{2}=0,40$ ).

No decorrer do desenvolvimento do experimento, morreram 11 moluscos do grupo Controle (taxa de mortalidade pré-maturidade sexual de $15 \%$ e pósmaturidade sexual de 13\%), 14 do grupo Dia longo (taxa de mortalidade pré-maturidade sexual de $3 \%$ e pós-maturidade sexual de 33\%) e 11 do grupo Dia curto (taxa de mortalidade pré-maturidade sexual de $13 \%$ e pós-maturidade sexual de $15 \%$ ). Não houve diferença significativa no número total de moluscos mortos entre os três tratamentos durante todo o período estudado $(\mathrm{H}=0,47 ; \mathrm{p}=0,79)$, antes da maturidade sexual $(H=2,60 ; p=0,27)$ e depois da maturidade sexual $(\mathrm{H}=3,41 ; \mathrm{p}=0,18)$.

\section{DISCUSSÃO}

O fotoperíodo e a temperatura são os principais fatores ambientais relacionados à reprodução dos moluscos terrestres (South, 1992; HommaY et al., 2001). Estes fatores podem regular a liberação de ovos ou filhotes, uma vez que os mecanismos endócrinos que influenciam a reprodução são dependentes de estímulos externos (WAYNE, 2001). Pesquisas mostraram que o fotoperíodo pode provocar alterações em parâmetros reprodutivos, como o tempo gasto para o alcance da maturidade sexual, a fecundidade e a viabilidade de ovos (Hommay et al., 2001).

As pesquisas sobre influência do fotoperíodo no ciclo de vida de gastrópodes terrestres foram feitos, na maior parte, com Helix aspersa Müller, 1774 (Helicidae), provavelmente por ser essa uma espécie de interesse econômico (BAILEy, 1981; Enée et al., 1982; Gomot et al., 1982; Bonnefoy-Claudet et al., 1983; Le GuHENNEC \& Daguzan, 1983; LaURent et al., 1984; Gomot \& Gomot, 1985; Griffond \& Vincent, 1985; Bonnefoy-Claudet \& Deray, 1987; Deray \& Laurent, 1987; Gomot et al., 1989). Tais estudos também foram conduzidos com Bradybaena similaris (Férussac, 1821) (Xanthonychidae) (GARCIA \& Pinheiro, 2007), Achatina achatina (Linnaeus, 1758) (Achatinidae) (HodAsI, 1982), Deroceras reticulatum (Müller, 1774) (Agriolimacidae) (Henderson \& Pelluet, 1960), Limax maximus Linnaeus, 1758 (Limacidae) (Sokolove \& Mccrone, 1978; Mccrone et al., 1981; Melrose et al., 1983), Helix pomatia Linnaeus, 1758 e Cepaea nemoralis (Linnaeus, 1758) (Helicidae) (HUNTER \& Stone, 1986; Gомот, 1990).

Em D. muibum observamos que a diminuição do número de horas de luz no dia favoreceu o aumento do número de eventos reprodutivos e de filhotes (no total, por evento reprodutivo e por molusco parental) e a diminuição dos intervalos entre os eventos sucessivos. Além disso, indivíduos de ambos os tratamentos com fotoperíodo artificial alcançaram a maturidade mais rapidamente do que aqueles do tratamento com fotoperíodo natural. Portanto, as condições controladas na estufa para B.O.D., como temperatura e umidade relativa do ar, favoreceram o início da atividade reprodutiva e a diminuição do comprimento do dia teve um efeito benéfico ainda maior sobre os demais parâmetros reprodutivos. Esses dados contrastam com os de HоммаY et al. (2001) que verificaram o contrário para a espécie Limax valentianus (Férussac, 1823) (Limacidae), observando maior produção de ovos em dias longos (16 horas de luz e 8 horas de ausência de luz), do que em dias curtos ( 12 horas de luz e 12 horas de ausência de luz).

Gомот et al. (1989) também verificaram um melhor padrão reprodutivo em Helix aspersa, apresentando maior fecundidade em dias longos ( 18 horas de luz e 6 horas de escuro) e temperatura de $20^{\circ} \mathrm{C}$. Esses autores observaram que em dias curtos ( 8 horas de luz e 16 horas de escuro) e temperatura de $20^{\circ} \mathrm{C}$, a ovipostura foi parcialmente inibida, e quando a temperatura era reduzida para $15^{\circ} \mathrm{C}$, a ovipostura foi inibida por completo. A temperatura pode afetar o ciclo de vida dos moluscos terrestres ao causar alterações na reprodução, no desenvolvimento embrionário, nas taxas de eclosão e desenvolvimento dos filhotes, na taxa de crescimento, no comportamento e na sobrevivência, sendo um fator decisivo na biologia desses animais (FURTADO et al., 2004).

Em D. muibum houve relação direta entre o comprimento médio da concha e o número médio de filhotes nos três tratamentos, de modo que a fecundidade aumenta com o tamanho do corpo, como também verificado em Cepaea nemoralis (Wolda \& Kreulen, 1973). A espécie também apresentou, em todos os tratamentos, estratégia reprodutiva iterópara, ou seja, o investimento da energia em mais de um ciclo reprodutivo, não tendo o fotoperíodo quaisquer efeitos sobre esses padrões. Segundo Gомот 
et al. (1989), os efeitos do fotoperíodo na reprodução devem-se principalmente à alteração da maturação dos gametas e síntese de albúmen, provavelmente devido à alteração hormonal.

Embora não tenha sido registrada na literatura sazonalidade reprodutiva nessa espécie, sabe-se que muitas vezes ela está relacionada a fatores como temperatura, pluviosidade e fotoperíodo (UDAKA et al., 2007). Em Megalobulimus abbreviatus (Bequaert, 1948) (Megalobulimidae), a atividade reprodutiva ocorre principalmente na primavera, provavelmente favorecida por mudanças ambientais características dessa estação, como aumento do comprimento do dia e da temperatura (Horn et al., 2005). Vale ressaltar que no presente trabalho as condições de laboratório, como alimento e umidade do substrato constantes, podem ter favorecido a liberação de filhotes durante todo o período de estudo.

O comprimento da concha de D. muibum diferiu entre os moluscos dos três tratamentos, ao longo do período de estudo, tendo os espécimes do grupo Controle apresentado menor tamanho que os dos grupos em condições artificiais, e estes últimos diferido entre si ao final do experimento, quando os espécimes do grupo de menor comprimento do dia demonstrou maior crescimento. Apesar disso, as diferentes condições a que os indivíduos foram expostos não influenciaram a mortalidade que foi baixa antes e após a maturidade sexual, as taxas e o padrão de crescimento indeterminado. Esse padrão se deve aos moluscos investirem sua energia no crescimento antes de alcançarem a maturidade sexual e, ao alcançarem, investirem a maior parte de sua energia na reprodução, diminuindo assim o crescimento (HeLler, 2001), como encontrado nos subulinídeos Subulina octona (Brugüière, 1792) (BESSA \& AraúJo, 1995a) e Leptinaria unilamellata (d'Orbigny, 1835) (Almeida \& Bessa, 2001a; Carvalho et al., 2009), e em espécies de outras famílias de moluscos pulmonados terrestres, como Bradybaena similaris (ALMEIDA \& BESSA, 2001b; Carvalho et al., 2008) e Bulimulus tenuissimus (d'Orbigny, 1835) (Bulimulidae) (SiLva et al., 2008), e Habroconus semenlini (Moricand, 1845) (Euconulidae) (SiLva et al., 2009).

Os moluscos subulinídeos, como D. muibum, foram, em grande maioria, descritos com base apenas na morfologia da concha, porém nem sempre isso é possível devido às semelhanças entre espécies (Снон et al., 2006) e variações entre populações de uma mesma espécie (CALDEIRA et al., 2000; Minton et al., 2008). Estudos de variações nas medidas da concha podem resultar na constatação de diferentes padrões morfométricos, permitindo inferir sobre características do animal, como forma do corpo e padrão de crescimento (CHIU et al., 2002).

Ao final do experimento, o crescimento em comprimento da concha e demais medidas morfométricas obtidas permitiu verificar que $D$. muibum apresentou diferença em todas as variáveis lineares analisadas e em três das cinco razões entre essas variáveis, permitindo comprovar a eficiência de estudos da morfometria citada por CHIU et al. (2002). Entretanto, não foi possível distinguir conquiliomorfometrias características para os três tratamentos, com a distinção de morfotipos, pois os valores das variáveis e razões apenas aumentavam de forma paralela nos três tratamentos, não alterando, assim, a forma da concha, sempre alongada e cônica. Esse formato se deve provavelmente ao valor relativamente alto da razão entre o comprimento e a largura da concha, além das dimensões da volta corporal, expressas pela razão entre o comprimento da volta corporal e a largura da concha e pela proporção relativa entre a volta corporal e a espira. Entretanto, fatores abióticos como características do ambiente podem atuar na definição da morfologia da concha. Moluscos de habitats úmidos e protegidos, como $D$. muibum, tendem a ter conchas com espiras altas e aberturas maiores (embora a abertura da concha de D. muibum seja pequena), enquanto os de locais secos e sem proteção, como áreas de alteração antrópica, tendem a ter conchas com espiras baixas e aberturas estreitas (CHIBA \& DAVISON, 2007). Isso pode estar relacionado à menor retenção de umidade e temperaturas maiores nessas áreas, já que uma maior abertura da concha leva a uma maior exposição do tegumento e, consequentemente, à desidratação. Além disso, o maior comprimento expõe o animal a predadores (SAntos \& Monteiro, 2001).

Por outro lado, os grupos tratados com fotoperíodo artificial praticamente não diferiram entre si, demonstrando que não foi o fotoperíodo que influenciou o crescimento e a morfometria, mas o fato de tais animais encontraremse sob condições controladas, constantes e satisfatórias de temperatura e umidade relativa do ar. Para o melhor entendimento de como esses aspectos físicos influenciam os diversos fatores biológicos e morfológicos dos moluscos, é necessário o estudo da influência de diferentes temperaturas e umidades relativas do ar, assim como a influência da umidade do solo, sobre tais fatores.

As variações morfológicas observadas são provavelmente determinadas geneticamente, porém estudos de herança genética envolvendo esses animais são escassos na literatura científica (BACKELJAU et al., 2001; PINCEEL et al., 2005; ANDERSON, 2007; ARMBruster et al., 2007). Uma vez que os moluscos terrestres apresentam locomoção reduzida, ocorre baixo fluxo genético entre populações separadas geograficamente, fazendo com que apresentem uma constituição gênica distinta e em muitos casos sejam adaptadas às condições ambientais nas quais estão inseridas (Woodruff, 1980; Pfenninger \& Posada, 2002).

Agradecimentos. Agradecemos à Coordenação de Aperfeiçoamento de Pessoal de Nível Superior (CAPES) pelas bolsas concedidas.

\section{REFERÊNCIAS BIBLIOGRÁFICAS}

Almeida, M. N. \& Bessa, E. C. A. 2001a. Estudo do crescimento e da reprodução de Leptinaria unilamellata (d'Orbigny) (Mollusca, Subulinidae) em laboratório. Revista Brasileira de Zoologia 18(4):1107-1113. 
2001b. Estudo do crescimento e da reprodução de Bradybaena similaris (Férussac) (Mollusca, Xanthonychidae) em laboratório. Revista Brasileira de Zoologia 18(4):1115-1122.

Anderson, T. K.; Weaver, K. F. \& Guralnick, R. P. 2007. Variation in adult shell morphology and life-history traits in the land snail Oreohelix cooperi in relation to biotic and abiotic factors. Journal of Molluscan Studies 73:129-137.

Armbruster, G. F. J.; Hofer, M. \& Baur, B. 2007. Effect of cliff connectivity on the genetic population structure of a rock-dwelling land snail species with frequent self-fertilization. Biochemical Systematics and Ecology 35:325-333.

Asami, T. 1999. Effects of oviposition substrate on lifetime fecundity of terrestrial pulmonate Bradybaena similaris. Journal of Conchology 36:1-9.

Backeljau, T.; Baur, A. \& Baur, B. 2001. Population and conservation genetics. In: BARKER, G. M. eds. The biology of terrestrial molluses. New York, CABI Publishing. 558p.

BAILEY,S.E.R. 1981.Circannual and circadianrhythms in the snail Helixaspersa Müller and the photoperiodic control of annual activity and reproduction. Journal of Comparative Physiology 142:89-94.

Bessa, E. C. A. \& Araújo, J. L. B. 1995a. Oviposição, tamanho de ovos e medida do comprimento da concha em diferentes fases do desenvolvimento de Subulina octona (Brugüière) (Pulmonata, Subulinidae) em condições de laboratório. Revista Brasileira de Zoologia 12(3):647-654.

1995b. Ocorrência de autofecundação em Subulina octona (Brugüière) (Pulmonata, Subulinidae) em condições de laboratório. Revista Brasileira de Zoologia 12(3):719-723.

Bonnefoy-Claudet, R. \& Deray, A. 1987. Modalités de reproduction de l'escargot Helix aspersa maxima en fonction de la photophase, comparaison avec Helix aspersa aspersa. Haliotes 16:69-75.

BonNefoy-Claudet, R.; Deray, A. \& Gomot, L. 1983. Action de lumières de longueurs d'onde différentes sur la reproduction de l'escargot Helix aspersa. Comptes Rendus des Séances de la Société de Biologie et de ses Filiates 177(4):504-512.

Caldeira, R. L.; Vidigal, T. H. D. A.; Matinella, L.; Simpson, A. J. G. \& CARVALHO, O. S. 2000. Identification of planorbids from Venezuela by polymerase chain reaction amplification and restriction fragment length polymorphism of internal transcriber spacer of the RNA ribosomal gene. Memórias do Instituto Oswaldo Cruz 95(2):171177.

Carvalho, C. M.; Bessa, E. C. A. \& D’Ávila, S. 2008. Life history strategy of Bradybaena similaris (Férussac, 1821) (Mollusca, Pulmonata, Bradybaenidae). Molluscan Research 28(3):171-174

Carvalho, C. M.; Silva, J. P.; Mendonça, C. L. F.; Bessa, E. C. A. \& D’ÁvilA, S. 2009. Life history strategy of Leptinaria unilamellata (d'Orbigny, 1835) (Mollusca, Pulmonata, Subulinidae). Invertebrate Reproduction \& Development 53(4):211-222.

ChiBA, S. \& Davison, A. 2007. Shell shape and habitat use in the northwest pacific land snail Mandarina polita from Hahajima, Ogasawara: current adaptation or ghost of species past? Biological Journal of the Linnean Society 91:149-159.

Chiv, Y. W.; Chen, H. C.; Lee, S. C. \& Chen, C. A. 2002. Morphometric analysis of shell and operculum variations in the viviparid snail, Cipangopaludina chinensis (Mollusca: Gastropoda), in Taiwan. Zoological Studies 41(3):321-331.

Снон, M. S.; YAP, C. K.; TAN, S. G. \& JAMBARI, H. A. 2006. Morphological and allozyme studies of small terrestrial snails (Opeas sp., Subulina sp. and Huttonella bicolor) collected from Peninsular Malaysia. Genetika 42(1):49-57.

D’Ávila, S. \& BeSSA, E. C. A. 2005a. Influência do substrato sobre a reprodução de Subulina octona (Brugüière) (Mollusca, Subulinidae), sob condições de laboratório. Revista Brasileira de Zoologia 22(1):197-204.

2005b. Influência do substrato sobre o crescimento de Subulina octona (Brugüière) (Mollusca, Subulinidae), sob condições de laboratório. Revista Brasileira de Zoologia 22(1):205-211.

2005c. Influência de diferentes substratos e umidade sobre o crescimento e número de ovos produzidos por Subulina octona (Brugüière) (Mollusca, Subulinidae), sob condições de laboratório. Revista Brasileira de Zoologia 22(2):349-353.
D’Ávila, S.; Dias, R. J. P.; Bessa, E. C. A. \& DAemon, E. 2004. Resistência à dessecação em três espécies de moluscos terrestres: aspectos adaptativos e significado para o controle de helmintos. Revista Brasileira de Zoociências 6(1):115-127.

Deray, A \& LAURENT, J. 1987. Lumière et dynamique de croissance chez l'escargot Helix aspersa maxima (gros gris) en conditions hors sol contrôlées. Comptes Rendus des Séances de la Société de Biologie et de ses Filiates 181(2):179-186.

Dimitrieva, E. F. 1975. The influence of temperature and moisture of the upper soil layer on the hatching intensity of the slug Deroceras reticulatum Müller. Malacological Review 10:32-45.

Enée, J.; Bonnefoy-Claudet, R. \& Gomot, L. 1982. Effet de la photopériode artificielle sur la reproduction de l'escargot Helix aspersa Müller. Comptes Rendus de l'Académie des Sciences 294:357-360.

Furtado, M. C. V.; Bessa, E. C. A. \& Castañon, M. C. M. N. 2004. Ovoteste de Bradybaena similaris (Férussac, 1821) (Mollusca, Xanthonychidae): histologia e produção de gametas. Revista Brasileira de Zoociências 6:7-18.

Garcia, T. A. \& Pinheiro, J. 2007. The photoperiod may modulate the carbohydrate metabolism of Bradybaena similaris (Férussac, 1821) (Mollusca, Bradybaenidae). Revista Brasileira de Zoociências 9(1):7-12.

Gомот, А. 1990. Photoperiod and temperature interaction in the determination of reproduction of the edible snail, Helix pomatia. Journal of Reproduction and Fertility 90:581-585.

Gomot, L.; EnÉe, J. \& LAuRent, J. 1982. Influence de la photopériode sur la croissance pondérale de l'escargot Helix aspersa Müller en milieu contrôlé. Comptes Rendus de l'Académie des Sciences 294:749-752.

Gомот, P. \& Gомот, L. 1985. Action de la photopériode sur la multiplication spermatogoniale et la reproduction de l'escargot Helix aspersa. Bulletin de la Société Zoologique de France 110:445-459.

Gомот, P.; Gомот, L. \& Griffond, B. 1989. Evidence for a light compensation of the inhibition of reproduction by low temperatures in the snail Helix aspersa. Ovotestis and albumen gland responsiveness to different conditions of photoperiods and temperatures. Biology of Reproduction 40:1237-1245.

Griffond, B. \& Vincent, C. 1985. Etude de l'activité des corps dorsaux de l'escargot Helix aspersa Müller au cours des phases physiologiques de la vie adulte et sous différentes photopériodes. International Journal of Invertebrate Reproduction and Development 8(1):27-37.

HAUSDORF, B. 2006. Is the interspecific variation of body size of land snails correlated with rainfall in Israel and Palestine? Acta Oecologica 30(3):374-379.

Heller, J. 2001. Life history strategies. In: Barker, G. M. eds. The biology of terrestrial molluscs. New York, CABI Publishing. 558p.

Henderson, N. E. \& Pelluet, D. 1960. The effect of visible light on the ovotestis of the slug Deroceras reticulatum Müller. Canadian Journal of Zoology 38(1):173-178.

Hodasi, J. K. M. 1982. The effects of different light regimes on the behavior and biology of Achatina (Achatina) achatina (Linné). Journal of Molluscan Studies 48:283-293.

Hommay, G; Kienlen, J. C.; Gertz, C. \& Hill, A. 2001. Growth and reproduction of the slug Limax valentianus Férrusac in experimental conditions. Journal of Molluscan Studies 67:191-207.

Horn, A. C.; AchaVAL, A. \& ZanCAN, D. M. 2005. The annual reproductive cycle of the snail Megalobulimus abbreviatus (Bequaert, 1948) (Gastropoda, Pulmonata). Brazilian Journal of Biology 65(3):459467.

Hunter, R. D. \& Stone, L. M. 1986. The effect of artificial photoperiod on growth and reproduction in the land snail Cepaea nemoralis. International Journal of Invertebrate Reproduction and Development 9:339-344.

Laurent, J.; Deray, A. \& Grimard, A. M. 1984. Influence de la photopériode, du degré d'hétérogénéité de la population sur la dynamique de croissance et la maturation sexuelle de l'escargot Helix aspersa. Comptes Rendus des Séances de la Société de Biologie et de ses Filiates 178(4):421-441.

Le Guhennec, M. F. \& Daguzan, J. 1983. Rôle de la lumière sur la reproduction de l'escargot petit-gris, Helix aspersa Müller. Comptes Rendus de l'Académie des Sciences 297:141-144. 
LüsIs, O. 1966. Changes induced in the reproductive system of Arion ater rufus L. by varying environmental conditions. Proceedings of the Malacological Society of London 37:19-26.

McCrone, E. J.; Van Minnen, J. \& SoKolove, P. G. 1981. Slug reproductive maturation hormone: in vivo evidence for long-day stimulation of secretion from brains and cerebral ganglia. Journal of Comparative Physiology 143(3):311-315.

Melrose, G. R.; O’Neill, M. C. \& Sokolove, P. G. 1983. Male gonadotrophic factor in brain and blood of photoperiodically stimulated slugs. General and Comparative Endocrinology 52:319-328.

Minton, R. L.; Norwood, A. P. \& Hayes, D. M. 2008. Quantifying phenotypic gradients in freshwater snails: a case study in Lithasia (Gastropoda: Pleuroceridae). Hydrobiologia 605:173-182.

Mota, G. G. \& Almeida, M. N. 2012. Conquiliomorfometria, crescimento alométrico e ciclo de vida de Dysopeas muibum Marcus \& Marcus, 1968 (Pulmonata, Subulinidae) em condições de campo. Revista Eletrônica de Biologia 5(2)83-99.

Pfenninger, M. \& Posada, D. 2002. Phylogeographic history of the land snail Candidula unifasciata (Helicellinae, Stylommatophora): fragmentation, corridor migration and secondary contact. Evolution 56(9): 1776-1778.

Pilate, V. J.; Chicarino, E. D.; Daniel, P. A.; Santos, E. O.; Silva, L. C. \& Bessa, E. C. A. 2013. Estudo comportamental da agregação e preferência por sítio de repouso de moluscos subulinídeos em laboratório. Revista Nordestina de Zoologia 7(1):16-26.

Pilate, V. J.; Silva, L. C. \& Bessa, E. C. A. 2013. Ciclo de vida de Dysopeas muibum (Mollusca, Subulinidae) em laboratório: efeito do isolamento sobre padrões biológicos e conquiliomorfométricos. Iheringia, Série Zoologia 103(4):350-356.

Pilate, V. J.; Silva, L. C.; Vargas, T.; Souza, B. A.; Chicarino, E. D. \& Bessa, E. C. A. 2012. Repertório comportamental e horário de atividade do molusco terrestre Dysopeas muibum Marcus \& Marcus, 1978 (Mollusca, Subulinidae) em laboratório. BioFar - Revista de Biologia e Farmácia 8(2):176-188.

Pinceel, J.; Jordaens, K.; Van Houtte, N.; Bernon, G. \& Backeljau, T. 2005. Population genetics and identity of an introduced terrestrial slug: Arion subfuscus s.l. in the north-east USA (Gastropoda, Pulmonata, Arionidae). Genetica 125:155-171.

Santos, S. B. \& Monteiro, D. P. 2001. Composição de gastrópodes terrestres em duas áreas do Centro de Estudos Ambientais e
Desenvolvimento Sustentado (CEADS), Vila Dois Rios, Ilha Grande, Rio de Janeiro, Brasil - um estudo-piloto. Revista Brasileira de Zoologia 18(1):181-190.

Silva, L. C.; Meireles, L. M. O.; Junqueira, F. O. \& Bessa, E. C. A. 2008. Development and reproduction in Bulimulus tenuissimus (Mollusca: Bulimulidae) in laboratory. Revista Brasileira de Zoologia 25(2):220-223.

Silva, L. C.; Meireles, L. M. O.; Vargas, T.; Junqueira, F. O. \& Bessa, E. C. A. 2009. Life history of the land snail Habroconus semenlini (Stylommatophora: Euconulidae) under laboratory conditions. Revista de Biologia Tropical 57(4):1217-1222.

Simone, L. R. L. 2006. Land and freshwater molluses of Brazil. São Paulo, Fapesp. 390p.

Sokolove, P. G. \& Mccrone, E. J. 1978. Reproductive maturation in the slug Limax maximus, and the effects of artificial photoperiod. Journal of Comparative Physiology 125(4):317-325.

South, A. 1992. A comparison of the life cycles of Deroceras reticulatum (Müller) and Arion intermedius Normand (Pulmonata: Stylommatophora) at different temperatures under laboratory conditions. Journal of Molluscan Studies 48:233-244.

Teles, H. M. S. \& Fontes, L. R. 2002. Implicações da introdução e dispersão de Achatina fulica Bowdich, 1882 no Brasil. Boletim do Instituto Adolfo Lutz 12:3-5.

Udaka, H.; Mori, M.; Goto, S. G. \& Numata, H. 2007. Seasonal reproductive cycle in relation to tolerance to high temperatures in the terrestrial slug Lehmannia valentiana. Invertebrate Biology 126(2):154-162.

WAREING, D. R. \& Bailey, S. E. R. 1985. The effects of steady and cycling temperatures on the activity of the slug Deroceras reticulatum. Journal of Molluscan Studies 51(3):257-266.

WAYNE, N. L. 2001. Regulation of seasonal reproduction in molluscs. Journal of Biological Rhythms 16(4):391-402.

Wolda, H. \& Kreulen, D. A. 1973. Ecology of some experimental populations the land snail Cepaea nemoralis (L.). II. Production and survival of eggs and juveniles. Netherlands Journal of Zoology 23(2):168-188

WoodRufF, D. S. 1980. Geographic differentiation and speciation in Cerion - a preliminary discussion of patterns and processes. Biological Journal of the Linnean Society 14:389-416. 